\title{
INTERCULTURALIDAD Y EDUCACIÓN BÁSICA. UN ESTUDIO EN UNA INSTITUCIÓN EDUCATIVA PERUANA
}

\author{
INTERCULTURALITY AND BASIC EDUCATION. \\ A STUDY MADE IN A PERUVIAN EDUCATIONAL INSTITUTE
}

\author{
Mitchell Alarcón Díaz ${ }^{1}$ \\ Henry Alarcón Díaz ${ }^{2}$ \\ Víctor Gutiérrez Cisneros ${ }^{3}$ \\ Sulma Quispe Rojas ${ }^{4}$
}

\section{RESUMEN}

La investigación tuvo como objetivo determinar las diferencias entre los niveles de interculturalidad según el género, el lugar de procedencia y la vivencia con las costumbres tradicionales de los alumnos del nivel secundaria de la Institución Educativa N. 157 "José Abelardo Quiñones" (Perú). Para tal fin se puso en práctica un estudio basado en un enfoque cuantitativo, con un método hipotético deductivo y con diseño transeccional descriptivo. A partir de la aplicación del instrumento de rasgos interculturales se concluye que existen diferencias significativas, con tendencia a un nivel fuerte, en el caso del género y la vivencia de las costumbres del alumnado.

\section{Palabras clave}

Costumbres tradicionales, escuela, género, identidad cultural, interculturalidad

\section{ABTRACT}

The research was aimed to determine the differences between the levels of multiculturalism according to gender, place of origin, and the living experience with the traditional customs of students of high school No. 157 "José Abelardo Quiñones" (Peru). To reach this goal, the researchers implemented a study based on a quantitative approach, with a hypothetico-deductive method and a descriptive cross-sectional design. From the application of the intercultural traits instrument, we conclude that there are significant differences, with a strong level tendency, in gender and customs living experiences of the students.

\section{Keywords}

Traditional customs, school, gender, cultural identity, interculturality.

\footnotetext{
Doctor en ciencias de la Educación por la Universidad Nacional de Educación Enrigue Guzmán y Valle (Perú). Autor de diversas publicaciones relacionadas con la teoría de la educación y la investigación científica.

2 Doctor en Educación por la Universidad de San Martín de Porres (Perú). Ha desarrollado el Posdoctorado de Comunicación, Educación y Cultura en la Universidad Santo Tomás, Bogotá, Colombia.

3 Magíster en Educación con Mención en Docencia y Gestión Educativa por parte de la Universidad César Vallejo (UCV) - Perú.

4 Magíster en Educación con Mención en Docencia y Gestión Educativa por parte de la Universidad César Vallejo (UCV) - Perú.
} 


\section{INTRODUCCIÓN}

Cuando se habla de interculturalidad, asumimos que este no es un acto que implique la tolerancia sino el reconocer la valía de las identidades dinámicas de los colectivos que coexisten en un espacio social. Servindi (2005) comenta que "La interculturalidad es un proceso de interrelación que parte de la reflexión del reconocimiento de la diversidad y del respeto a las diferencias" (p. 28). Otra lectura posible acerca del tema es que la interculturalidad puede ser concebida como una necesidad ya que, su efecto integrador, enriquece el escenario pluricultural en los que la diversidad forma parte de la esencia de las sociedades. En todo caso, en el escenario educativo la interculturalidad puede significar una oportunidad para promover procesos de intercambio y consensos de interrelación entre los actores educativos.

La propuesta intercultural en América Latina aparece como una opción válida frente a los lineamientos antiguos del sistema y que visualiza a la sociedad como un ente unicultural. De esta forma, en algunos países tales como México, Bolivia, Colombia, Perú, etc. se emprenden acciones con el fin de interrelacionar a los distintos colectivos existentes al interior de la sociedad.

Servindi (2005) comenta que, es en este escenario en el que por ejemplo, en la década de los 80 se introduce el enfoque de interculturalidad en proyectos de educación bilingüe lo cual es reforzado a través del Encuentro sobre Educación Indígena (Oaxaca, México, 1983).

Es ya en la década de los 90 es cuando gran parte de los países de América Latina aceptan su condición de sociedades pluriculturales. Esto se constituyó en un hecho sumamente importante ya que, implicaba el establecer lineamientos de políticas, por ejemplo educativa, con el fin de promover la interculturalidad a través de dimensiones tales como el lenguaje, la cosmovisión, la danza, la música, la vestimenta, el tema de género, etc. El espacio de influencia creado fue tan amplio que abarcó los diferentes niveles y modalidades extendiéndose también a nivel rural y urbano.

Lo acontecido en los últimos años en el Perú a través del reconocimiento a los patrimonios culturales (lugares, fiestas costumbristas, gastronomía, música, etc.) ha hecho reflexionar acerca de cuan diversas pueden ser las relaciones y los significados que como sociedad le podemos dar al fenómeno intercultural: la autoestima, el reconocimiento y la comprensión de la diversidad cultural existente.

Por ejemplo, en lo que se refiere al tema del género, las Naciones Unidas proponen desarrollar políticas multisectoriales que favorezcan el reconocimiento de los derechos de los hombres y de las mujeres desde mediados de la década de los 70 . Al respecto, Rodríguez e Iturmendi (2013) mencionan “... La interculturalidad con enfoque de género parte del reclamo de los derechos culturales, de la multiculturalidad como hecho social y del derecho a la diversidad cultural, considerando la desigualdad de género" (p. 35).

Así, se intenta que a nivel de los entes, por ejemplo las instituciones educativas, se incorpore la perspectiva de género a través de las diferentes manifestaciones, como por ejemplo, las reglas de convivencia, la orientación educativa, etc. A través de la interculturalidad, la visión de género puede presentarse como un ingrediente que aporte al intercambio y la apertura de las manifestaciones culturales con la que conviven las personas y de esa forma favorecer el reconocimiento de las diferencias para la construcción de un espacio dialógico respetuoso.

Otro caso que podemos mencionar es el relacionado con el lugar de procedencia. El centralismo del Estado, representado entre otros aspectos por la concentración del desarrollo de la economía en las grandes ciudades, ha provocado ciertas consecuencias. Una de ellas es la movilidad de las personas. Las familias deciden migrar hacia las urbes con el fin de encontrar un espacio de mejora personal y familiar. Así encontramos diversas identidades culturales que coexisten en un mismo espacio geográfico. 
Acerca del lugar de procedencia, Alamos y Furnaro (2013) comentan que:

El espacio, como un producto social, está siempre relacionado a la acción humana y sujeto a cambios, ya que su constitución depende de las prácticas que individuos y grupos lleven a cabo en el día a día. El espacio es creado por la realidad social y es una construcción antes que una realidad externa sobre la cual los individuos y las cosas se ubican. (p. 65)

En la institución donde se realizó este estudio se encontró que un porcentaje importante de los alumnos provenían de diversos lugares del país. Esto genera un espacio de encuentro pluricultural el cual podría enriquecer las intenciones interculturales de la propuesta educativa de la escuela.

Un caso más por considerar es el de la vivencia de las costumbres. La práctica de las manifestaciones costumbristas es una valiosa esencia de lo que algunas personas denominarían cultura (la gastronomía, el arte, la historia, la religión, etc.). Estas costumbres se han formado en las familias y en las comunidades a partir del legado de los pobladores antiguos. Hoy en día, y a pesar de que existen acentuados procesos de migración de los pueblos a las ciudades, las personas intentan mantener viva las costumbres propias de su región, de su pueblo. Eso ocurre cuando se observa superficialmente que los alumnos y padres de familia participan en las actividades escolares. Acerca de la práctica de las costumbres en la escuela, Palacios de Torres (2009) menciona que en ellas "se aspira a favorecer una integración de todos los alumnos procedentes de diferentes culturas, creyentes de distintas religiones y formados en valores familiares $y$ en costumbres muy diversas" (p. 2).

Por todo ello, hablar de identidad cultural es referirnos a la identificación, caracterización $y$ al sentido de pertenencia con relación a un conjunto de manifestaciones propias de un determinado lugar que consideramos como nuestro.

\section{MATERIAL Y MÉTODO}

Esta investigación se basó en un enfoque cuantitativo. Según Hernández, Fernández y Baptista (2014), este enfoque "utiliza la recolección de datos para probar hipótesis con base en la medición numérica y el análisis estadístico, con el fin de establecer pautas de comportamiento y probar teorías" (p. 4). El método empleado fue el hipotético deductivo. Acerca de ello, Fontaines (2012) comenta que en este proceso:

el investigador inicia su travesía en sus esquemas preconcebidos del mundo, $y$ mediante deducción, llega a establecer la validación de las teorías planteadas. En tal sentido, formula hipótesis teóricas, y se confrontan con las teorías implícitas en dichas hipótesis (por aquello de los esquemas preconcebidos). Seguidamente, se produce la confrontación de estas con los datos observacionales, para luego construir el modelo teórico y por último, someterlo a validación mediante el criterio de la falsación. (p. 47)

El diseño investigativo propuesto fue el transeccional descriptivo. Este tipo de diseños "indagan la incidencia de las modalidades, categorías o niveles de una o más variables en una población" (Hernández et al., 2014, p. 155).

Las unidades muestrales pertenecen a la Institución Educativa (I.E.) N. ${ }^{\circ} 157$ José Abelardo Quiñones ubicado en el distrito de San Juan de Lurigancho (Perú). Esta muestra estuvo conformada por 236 alumnos del nivel secundaria. Estos alumnos poseen características culturales diversas. Estas se han ido consolidando a través del tiempo a través de la influencia del entorno cercano (familiares, amigos, etc.). Así, la confluencia pluricultural existente provoca eventualmente algunas tensiones en el espacio educativo.

El instrumento de recolección de datos empleado en este estudio fue el Cuestionario de Identidad Intercultural, el mismo que considera dimensiones tales como la identidad 
cultural, el género, el lugar de procedencia y la vivencia de las costumbres. Este instrumento fue validado por los docentes de la Universidad César Vallejo (Perú). Para el análisis descriptivo, se procesaron los datos con el software SPSS v. 20, se aplicó la prueba de U de Mann-
Whitney, las tablas de contingencia y el gráfico de barras por tratarse de una prueba no paramétrica. Para la prueba de hipótesis se ha utilizado la prueba Kruskal-Wallis, dado que este estadístico es apropiado para medir los niveles de la variable.

Tabla 1.

Distribución de la muestra de la población de estudio

\begin{tabular}{lcccccc}
\hline & & Varones & & Mujeres & & Total \\
\hline Grados & $\mathrm{F}$ & $\%$ & $\mathrm{f}$ & $\%$ & $\mathrm{f}$ & $\%$ \\
\hline 1ro & 32 & $13.56 \%$ & 24 & $10.17 \%$ & 56 & $23.73 \%$ \\
2do & 26 & $11.02 \%$ & 28 & $11.86 \%$ & 54 & $22.88 \%$ \\
3ro & 24 & $10.17 \%$ & 30 & $12.71 \%$ & 54 & $22.88 \%$ \\
4to & 7 & $2.97 \%$ & 35 & $14.83 \%$ & 42 & $17.80 \%$ \\
5to & 11 & $4.66 \%$ & 19 & $8.05 \%$ & 30 & $12.71 \%$ \\
Total & 100 & $42.38 \%$ & 136 & $57.62 \%$ & 236 & $100 \%$ \\
\hline
\end{tabular}

\section{RESULTADOS Y DISCUSIÓN}

Tabla 2.

Identidad cultural

\begin{tabular}{lcc}
\hline Niveles de Identidad cultural & Frecuencia & Porcentaje \\
\hline Débil & 5 & $2.1 \%$ \\
Moderado & 120 & $50.8 \%$ \\
Fuerte & 111 & $47.1 \%$ \\
Total & 236 & $100 \%$ \\
\hline
\end{tabular}

La Tabla N. 2 muestra solo un $2.1 \%$ de los estudiantes con identidad cultural en el nivel débil, así mismo un $50.8 \%$ de ellos asumen la identidad cultural en el nivel moderado y un $47.7 \%$ tienen una identidad cultural fuerte.
Los resultados revelan que los estudiantes de la Institución Educativa $\mathrm{N}^{\circ} 157$ tienen una identidad cultural moderada con tendencia a un nivel fuerte.

Tabla 3.

Identidad de género

\begin{tabular}{lcccccc}
\hline $\begin{array}{l}\text { Niveles de } \\
\text { Identidad }\end{array}$ & $\mathrm{f}$ & \multicolumn{2}{c}{ Varón } & \multicolumn{2}{c}{ Mujer } & \multicolumn{2}{c}{ Totales } \\
$\%$
\end{tabular}


La Tabla N. ${ }^{\circ} 3$, muestra solo un $2.1 \%$ de los estudiantes varones refieren tener identidad según género en el nivel débil, un $25,4 \%$ de ellos en el nivel moderado y un $14,9 \%$ se ubican en el nivel fuerte. Con respecto a las estudiantes mujeres ninguna estudiante se ubica en el nivel débil, hay un $25,4 \%$ que refieren asumir una identidad según género en el nivel moderado, un $32,2 \%$ de estudiantes en el nivel fuerte. Los resultados revelan que los estudiantes de la Institución Educativa $\mathrm{N}^{\circ} 157$ tienen una identidad según género moderado con tendencia a un nivel fuerte con mayor ascendencia en las estudiantes mujeres.

Tabla 4.

Lugar de procedencia

\begin{tabular}{lcccccccc}
\hline $\begin{array}{l}\text { Niveles de } \\
\text { Identidad }\end{array}$ & \multicolumn{2}{c}{ Costa } & \multicolumn{2}{c}{ Sierra } & \multicolumn{2}{c}{ Selva } & \multicolumn{2}{c}{ Totales } \\
\hline Débil & 3 & $1,90 \%$ & 1 & $1,80 \%$ & 1 & $4,3 \%$ & 5 & $2,1 \%$ \\
Moderado & 82 & $52,60 \%$ & 23 & $40,30 \%$ & 15 & $65,2 \%$ & 120 & $50,8 \%$ \\
Fuerte & 71 & $45,50 \%$ & 33 & $57.90 \%$ & 7 & $30,5 \%$ & 111 & $47,1 \%$ \\
Total & 156 & $100 \%$ & 57 & $100 \%$ & 23 & $100 \%$ & 236 & $100 \%$ \\
\hline
\end{tabular}

En la Tabla N. ${ }^{\circ} 4$ se aprecia que solo un total de $2,1 \%$ de estudiantes procedentes de la costa, sierra y selva refieren asumir una identidad según el lugar de procedencia en el nivel débil, un $50,8 \%$ en el nivel moderado, finalmente un $47,1 \%$ en el nivel fuerte. Por lo tanto los resultados muestran que los estudiantes de la Institución Educativa $\mathrm{N}^{\circ} 157$ asumen una identidad moderada según lugar de procedencia con tendencia a llegar a un nivel fuerte.

Tabla 5.

Vivencia con las costumbres

\begin{tabular}{lccccccc}
\hline \multirow{2}{*}{$\begin{array}{l}\text { Niveles de } \\
\text { Identidad }\end{array}$} & \multicolumn{2}{c}{ Vivencia } & \multicolumn{3}{c}{ Costumbrista } & \multicolumn{2}{c}{} \\
& $\mathrm{f}$ & SI & $\%$ & $\mathrm{f}$ & $\mathrm{N}$ & \multicolumn{2}{c}{ Totales } \\
\hline Débil & 1 & $0,8 \%$ & 4 & $3,9 \%$ & 5 & $\%$ \\
Moderado & 57 & $42,5 \%$ & 63 & $61,8 \%$ & 120 & $50,8 \%$ \\
Fuerte & 76 & $56,7 \%$ & 35 & $34,3 \%$ & 111 & $47,0 \%$ \\
Total & 134 & $100,0 \%$ & 102 & $100,0 \%$ & 236 & $100,0 \%$ \\
\hline
\end{tabular}

En la Tabla N. ${ }^{\circ} 5$ se aprecia que solo un $2,2 \%$ de estudiantes refieren asumir una identidad, según su vivencia con las costumbres en Lima, en el nivel débil; un $50,8 \%$ de ellos en un nivel moderado y un $47,0 \%$ de estudiantes un nivel fuerte. Según los resultados del estudio los estudiantes de la Institución Educativa $\mathrm{N}^{\circ} 157$ se ubican en un nivel de la identidad moderado con tendencia a un nivel fuerte, como resultado del estudiante, papá y/o mamá procedentes de los zonas urbano-marginales del Perú, y las actividades culturales que realiza la institución educativa: concurso de danzas, concurso de potajes típicos, etc.

La información obtenida en el presente estudio muestra datos y rasgos acerca de los niveles de interculturalidad según el género, el lugar de procedencia y la vivencia con las costumbres tradicionales de los alumnos del nivel secundaria de la Institución Educativa $\mathrm{N}$. - 157 José Abelardo Quiñones. En la muestra de estudio, se hallaron diferencias significativas entre los niveles de la identidad según género 
y la vivencia con las costumbres. En lo que se refiere al lugar de procedencia, se muestra un nivel moderado.

Acerca de ello mencionaremos algunas ideas. De la Torre (2013) sostiene que la identidad responde al sentimiento de identificación, entre sí, de los miembros de una sociedad y que, como parte de dicha sociedad en su región, conservan costumbres y tradiciones de su lugar de origen.

En el Perú, los espacios urbanos se han incrementado $y$ se han constituido en lugares de encuentro multiculturales. En este escenario, referirse a interculturalidad es poder considerar la valía de las creencias, cosmovisiones, valores, etc. que son compartidas por un colectivo social. Esto explicaría los hallazgos del presente estudio: la relación significativa entre algunos rasgos: la identidad cultural, el tema de género, y la vivencia de las costumbres. En principio, la Institución Educativa $\mathrm{N}^{\circ} 157$ se ha convertido en una realidad intercultural ya que en ese espacio institucional se recrea la comunicación e interacción entre los integrantes de la comunidad. Al respecto, un reflexión de Leiva (2010) considera que:

Por todo ello, el centro educativo intercultural, la escuela intercultural, debe ser un espacio privilegiado de vida cultura y comunitaria. Es decir, los colegios e institutos de un barrio deben constituirse en centros generadores de participación comunitaria, ser permeables y estar abiertos a conformar en ellos una cultura escolar diversa, compleja y dinámica. La escuela intercultural $e$ inclusiva debe apostar claramente por la transformación crítica de la realidad que le ha tocado vivir, $y$ debe servir como canal de diversas expresiones éticas y culturales. (p. 163).

Por otro lado, se asume como cultura el hecho de asignarle valor a lo que se entiende como propio. Acerca de ello Poblete (2006) comenta que "la cultura es un sistema pautado $e$ integrado de creencias, costumbres, valores $e$ instituciones, lo que hace ver que todo en ella esta interrelacionado y ordenado de acuerdo a las formas de ser y actuar que el grupo ha construido como posibles." (p. 38).

Así, los niveles significativos de relaciones interculturales hallados en esta investigación demuestran la necesidad de alejarnos de la tendencia homogeneizadora en el espacio educativo. Abre las puertas a una visión de diversidad en el que los propósitos y fines de la educación se reinventan constantemente. En ese sentido, acerca de la interculturalidad y la escuela, Walsh (2005) manifiesta que:
se refiere a complejas relaciones, negociaciones e intercambios culturales, y busca desarrollar una interacción entre personas, conocimientos $y$ prácticas culturalmente diferentes; una interacción que reconoce y que parte de las asimetrías sociales, económicas, políticas y de poder y de las condiciones institucionales que limitan la posibilidad [de] que el "otro" pueda ser considerado como sujeto con identidad, diferencia y agencia la capacidad de actuar. (p. 6).

\section{CONCLUSIONES}

Los resultados de la significancia permiten señalar que la interculturalidad a través de los niveles de la identidad cultural, género, procedencia y vivencia de las costumbres son significativas, moderado con tendencia a un nivel fuerte, como resultado del estudiante, papá y/o mamá procedentes de los zonas urbano-marginales del Perú, y las actividades educativo - culturales que realiza la institución educativa N. ${ }^{\circ}$ 157: concurso de danzas, concurso de potajes típicos, concurso de talentos en composición e interpretación de poemas en lenguas originarias andinas y los viajes de estudios a los lugares históricos de la localidad y regional.

El trabajo de campo realizado muestra que en la Institución Educativa $N^{\circ} 157$ del distrito de San Juan de Lurigancho de Lima y en el año 2014 , solo un $2.1 \%$ de los estudiantes refieren tener una identidad cultural en el nivel débil, un $50.8 \%$ en el nivel moderado y un $47.1 \%$ en un nivel fuerte. 
Es en este contexto en el que, al reflexionar, consideramos que los espacios interculturales al interior de las aulas se constituyen en oportunidades que permiten distanciamiento de los centrismos y que aportan a una confluencia de acciones basadas en el reconocimiento, la democracia y la solidaridad. 


\section{REFERENCIAS}

Alamos, V. y Furnaro, A. (2013) Repensando el lugar de origen: estudiantes de hogares Mapuche en Temuco y Santiago, en Isees N. ${ }^{\circ} 12$. Disponible en Internet http://dialnet.unirioja.es/servlet/ articulo? codigo $=4421636$.

De La Torre, C. (2013) Identidad, identidades y ciencias sociales contemporáneas; conceptos, debates y retos. Disponible en Internet http://www.psicologia-online.com/articulos/2008/05/ identidad_identidades_y_ciencias_sociales.shtml. Consultado el 12 de enero de 2015.

Fontaines, T. (2012) Metodología de la investigación. Pasos para realizar el proyecto de investigación. Venezuela: Júpiter.

Hernández, R., Fernández, C. y Baptista, P. (2014) Metodología de la investigación. México: McGraw-Hill/Interamericana.

Leiva, J. (2010) La educación intercultural entre el deseo y la realidad: reflexiones para la construcción de una cultura de la diversidad en la escuela inclusiva, en La Revista Docencia e Investigación N. ${ }^{\circ} 20,149-182$.

Poblete, R. (2006) Educación intercultural: teorías, políticas y prácticas. La migración peruana en el Chile de hoy. Nuevos escenarios y desafíos para la integración. España: Tesis-Universidad Autónoma de Barcelona.

Palacios de Torres, C. (2009) Interculturalidad, distintas culturas y distintas lenguas con un mismo objetivo: la comunicación, en Contribuciones a las Ciencias Sociales. Disponible en Internet www. eumed.net/rev/cccss/04/cpt.htm.

Rodríguez, E. y Iturmendi, A. (2013) Igualdad de género e interculturalidad: enfoques y estrategias para avanzar en el debate. Panamá: RBLAC-PNUD.

Servindi (2005) Interculturalidad: Desafío y proceso en construcción. Perú: Sinco.

Walsh, C. (2005) La interculturalidad en la educación. Perú: Minedu.

Fecha de recepción: 19 de abril 2015

Fecha de aceptación: 08 de junio 2015 